\title{
Test Results of the First Two Full-Length Prototype Quadrupole Magnets for the LHC Hi-Lumi Upgrade
}

\author{
J. Muratore, K. Amm, M. Anerella, G. Ambrosio, G. Apollinari, M. Baldini, R. Carcagno, G. Chlachidze, D. Cheng, S. Feher, P. \\ Joshi, P. Kovach, A. Marone, M. Marchevsky, V. Marinozzi, H. Pan, E. Ravaioli, G, Sabbi, H. Song, P. Wanderer
}

\begin{abstract}
The future high luminosity (Hi-Lumi) upgrade of the Large Hadron Collider (LHC) at CERN will include eight (plus two spares) $8.4 \mathrm{~m}$-long cryostatted cold masses which will be components of the triplets for two LHC insertion regions. Each cold mass will consist of two $4.2 \mathrm{~m}$ long $\mathrm{Nb}_{3} \mathrm{Sn}$ high gradient quadrupole magnets, designated MQXFA, with aperture $150 \mathrm{~mm}$ and operating gradient $\mathbf{1 3 2 . 6} \mathrm{T} / \mathrm{m}$, for a total of twenty magnets. Before assembling and testing the final cold masses at Fermilab, the twenty component quadrupoles will be tested first at the vertical superconducting magnet test facility of the Superconducting Magnet Division at Brookhaven National Laboratory (BNL), in superfluid He at $1.9 \mathrm{~K}$ and to $18.0 \mathrm{kA}$, to meet $\mathrm{LHC}$ operational requirements. The first two full-length prototype quadrupole magnets, MQXFAP1 and MQXFAP2, have been tested at BNL. This paper reports on the quench test and training results of these magnets, and also the retest of the first prototype, rebuilt and designated as MQXFAP1b. The test results of these magnets will be important for validating the MQXFA design.
\end{abstract}

Index Terms - LARP, AUP, Hi-Lumi, LHC, Nb33 Sn, superconducting magnets

\section{INTRODUCTION}

The future high luminosity (Hi-Lumi) upgrade of the Large Hadron Collider (LHC) at CERN will include eight $8.4 \mathrm{~m}$ long cryostatted cold masses (plus two spares) which will be components of the Q1 and Q3 elements of the triplets at two LHC insertion regions. Each cold mass will consist of two $4.2 \mathrm{~m}$ long (magnetic length) high gradient quadrupole magnets, designated MQXFAxx, with aperture $150 \mathrm{~mm}$ and nominal operating gradient $132.6 \mathrm{~T} / \mathrm{m}$, for a total of twenty magnets. The fabrication and testing of these magnets are the combined effort of three US DOE laboratories: Brookhaven National Laboratory (BNL), Fermilab, and Lawrence Berkeley National Laboratory (LBNL), which together comprise the Accelerator Upgrade Project (AUP). In order to achieve the high field gradient necessary, the magnet coils are wound with $\mathrm{Nb}_{3} \mathrm{Sn}$ superconductor cable, taking advantage of the high current densities possible, especially at $1.9 \mathrm{~K}$. These coils must go through extensive heat treatment to create the superconductor, then vacuum impregnation to harden the coils against motion of the cables during cooldowns, warmups, and powering. Because of the strain-sensitive behavior of $\mathrm{Nb}_{3} \mathrm{Sn}$ superconductor and the necessity to limit the thickness of ground insulation to accommodate required quench protection heaters,

This work was supported by the U.S. Department of Energy, Office of Science, Office of High Energy Physics, through the US LHC Accelerator Upgrade Project (AUP). these magnets are challenging to fabricate and test, and both mechanical and electrical issues are possible, and have occurred. For this reason, before assembling and testing the final cold masses at Fermilab, the component quadrupoles will be tested individually at the vertical superconducting magnet test facility of the Superconducting Magnet Division at BNL, in superfluid $\mathrm{He}$ at $1.9 \mathrm{~K}$ and 1 bar pressure, and to $18.0 \mathrm{kA}$, in accordance with LHC operational requirements. Data from extensive cold testing of short models at Fermilab and CERN have shown successful, though slow, quench training and excellent training memory over thermal cycles, when the magnet is warmed up and then cooled down again. The first two long prototypes MQXFAP1 (and a rebuilt version MQXFAP1b) and MQXFAP2 have been tested at BNL in a $6 \mathrm{~m}$ deep vertical test cryostat, and the results of the quench training for these three tests are discussed here.

\section{MAGNET DESCRIPTION}

Fig. 1 shows the cross-section of the MQXFA design, with four 2-layer coils in the preloading structure. For vertical tests of the individual magnets, only the aluminum shell, consisting of 8 segments, is included. The stainless steel He containment vessel shown in the drawing will be installed at Fermilab when assembling the cold mass containing two quadrupoles. For MQXFAP1, starting with the upper right quadrant in Fig. 1 and moving counterclockwise, the four coils are designated P02, P03, P04, and P05, which are slightly shorter coils and use different conductors, and so are named differently from all other coils. For MQXFAP2, the coils are 102, 104, 105 , and 106, which are of nominal design and length.

The main parameters important for testing of the MQXFA

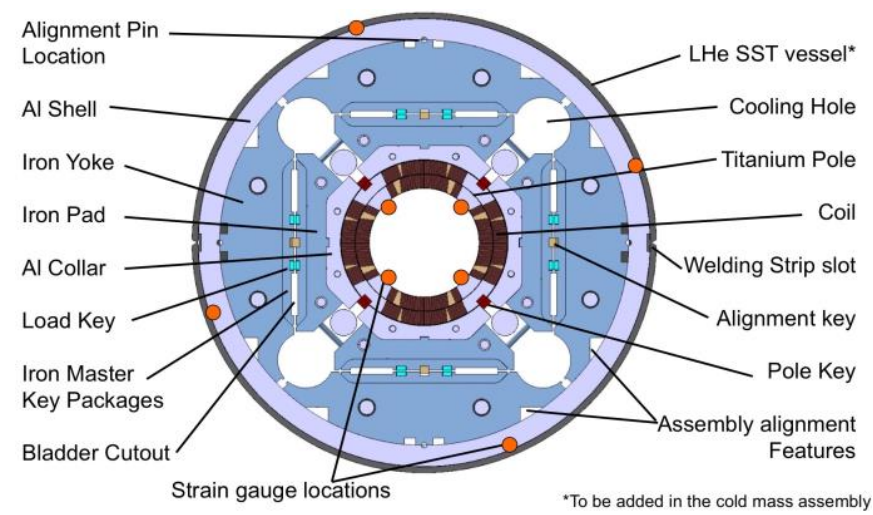

Fig. 1. MQXFA high gradient quadrupole cross-section.

Template version 8.0d, 22 August 2017. IEEE will put copyright information in this area See http://www.ieee.org/publications_standards/publications/rights/index.html for more information. 
TABLE I

MQXFA DESIGN AND TEST PARAMETERS

\begin{tabular}{ll}
\hline \hline \multicolumn{1}{c}{ Parameter } & \multicolumn{1}{c}{ Value } \\
\hline Coil inner aperture: & $150 \mathrm{~mm}$ \\
Coil magnetic length: & $4.2 \mathrm{~m}(4.0 \mathrm{~m})$ \\
Total length with end plates: & $5 \mathrm{~m}(\mathrm{nom})$ \\
Operational temperature and pressure: & $1.9 \mathrm{~K}$ and $1 \mathrm{bar}$ \\
LHC nominal operating current $(1.9 \mathrm{~K}):$ & $16.470 \mathrm{kA}$ \\
LHC ultimate operating current $(1.9 \mathrm{~K}):$ & $17.890 \mathrm{kA}$ \\
Conductor limit at $1.9 \mathrm{~K}:$ & $21.600 \mathrm{kA}$ \\
Conductor limit at 4.5 K: & $19.550 \mathrm{kA}$ \\
Nominal ramp rate: & $20 \mathrm{~A} / \mathrm{s}$ \\
Magnet inductance (1.9 K, $1 \mathrm{kA}):$ & $43.0 \mathrm{mH}(40.9 \mathrm{mH})$ \\
Magnet inductance $\left(1.9 \mathrm{~K}, \mathrm{I}_{\text {nom }}=16.5 \mathrm{kA}\right):$ & $34.4 \mathrm{mH}(32.8 \mathrm{mH})$ \\
Nominal stored energy (at $\left.\mathrm{B}_{\text {nom }}, \mathrm{I}_{\text {nom }}\right):$ & $4.67 \mathrm{MJ}$ \\
Ultimate stored energy (at $\left.\mathrm{B}_{\text {ult }}, \mathrm{I}_{\mathrm{ult}}\right)$ & $5.50 \mathrm{MJ}$ \\
Maximum allowed temperature at quench: & $250 \mathrm{~K} \approx 32(28) \mathrm{MIIts}$ \\
Maximum allowed voltage across magnet: & $1000 \mathrm{~V}$ \\
Dump resistor (energy extraction) options: & $30,37.5,50,75,150 \mathrm{~m} \Omega$ \\
Data sampling rate: & $10-100 \mathrm{MHz}$ \\
\hline
\end{tabular}

quadrupoles are listed in Table I. It should be noted that the magnetic length of MQXFAP1/P1b is $4.0 \mathrm{~m}$, and not the final design length of $4.2 \mathrm{~m}$. Due to decreased length, the inductance of MQXFAP1/P1b is less and is shown in parentheses. The actual length of both prototype structures and all production magnets (with endplates attached) is $5 \mathrm{~m}$, which the $\mathrm{He}$ vessel of the test cryostat at BNL was modified to accommodate. As shown in Table I, the nominal operating current is 16.470 A, and the so-called ultimate current is $17.890 \mathrm{~A}$, for a future upgrade of the LHC. These were the target currents to be reached during testing. It should also be noted that the maximum allowed hot spot temperature for training quenches is $250 \mathrm{~K}$. The corresponding quench integral $\left(\int \mathrm{I}^{2} \mathrm{dt}\right)$ value depends on the conductor used in each magnet. The MIIts value for MQXFAP1/P1b is shown in parentheses and is different because the conductor is different from the other magnets [1]. A higher temperature of $350 \mathrm{~K}$ was allowed only for the prototypes, after training was completed, and only during quench protection studies. Also, important to note is the high stored energy released during a quench.

\section{EXPERIMENTAL INSTRUMENTATION AND PRO- CEDURES}

Test procedures for the prototypes derive from an AUP specifications document [2], which is based on operational conditions of the magnets in the LHC triplets. Test parameters that must be met by the test facility are detailed in [3]; these include $1.9 \mathrm{~K}$ superfluid $\mathrm{He}$ at 1 bar vapor pressure, powering up to $24 \mathrm{kA}, 20 \mathrm{~A} / \mathrm{s}$ ramp rate for training, up to $160 \mathrm{~A} / \mathrm{s}$ for special tests, and the capability of He gas recovery at high and swiftly increasing pressures due to the large stored energy at high quench currents. Upgrades to the test facility were necessary to meet these requirements [4]-[6].

The MQXFA prototypes were instrumented with the following devices:

1) Voltage taps - main (fixed) taps for quench detection and auxiliary (configurable) taps for quench location.

2) Quench antenna with 16 elements, each having two sets of windings on a printed circuit board.

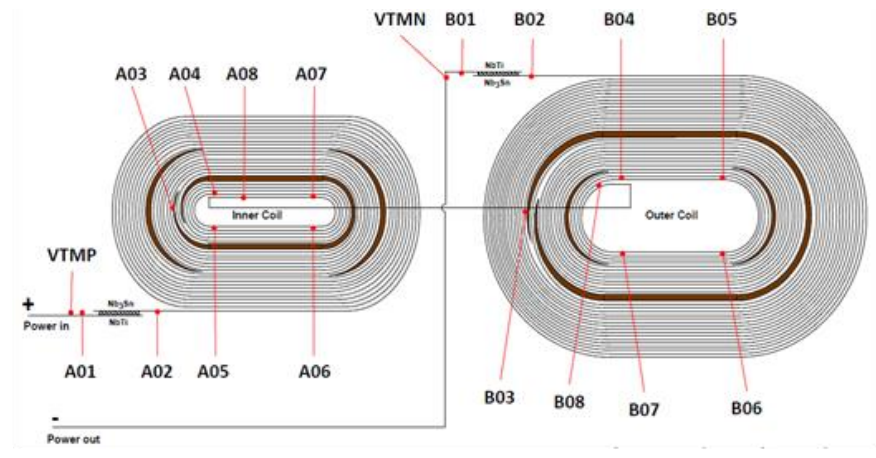

Fig. 2. MQXFA voltage tap configuration.

3) Strain gauges - two independent systems (Vishay and HBM) installed on coil poles, shells, and axial rods.

4) Rotating coil magnetic field measuring probe with $220 \mathrm{~mm}$ and $110 \mathrm{~mm}$ long windings.

5) Temperature, LHe level, and pressure sensors.

This paper is concerned with quench test results only; other testing operations and results are reported in [7]-[9]. Therefore, the instrumentation most relevant to this discussion are the voltage taps and signals produced by them during quenches. Fig. 2 shows the voltage tap schematic, a configuration used by all the AUP collaborating labs and CERN. Each layer of each coil has 8 taps which are located mainly on the pole turns, as can be seen. Additional taps, not shown, are located at splices between coils and on the leads.

Data acquisition was done with a fast data logger, sampling at $10 \mathrm{kHz}-100 \mathrm{kHz}$, depending on requirements, and a slow data logger which sampled at $1 \mathrm{~Hz}$ during quench and other ramp tests. Two FPGA-based digital quench detector (QD) systems were inputted with the half magnet voltage difference, total magnet voltage minus $\mathrm{L}(\mathrm{dI} / \mathrm{dt})$, and voltages of special tap sections, such as splices and superconducting leads [6].

Quench protection during testing of the prototypes was provided by the following systems and settings:

1) energy extraction using $37.5 \mathrm{~m} \Omega$ dump resistance;

2) quench protection heaters at $465 \mathrm{~V}, 190 \mathrm{~A}$, and $12.4 \mathrm{mF}$ to achieve the necessary power density;

3) Coupling Loss Induced Quench (CLIQ) system [10], set to $500 \mathrm{~V}$ and $40 \mathrm{mF}$.

Cooldown of a magnet under test consisted of three stages:

1) cooldown to $100 \mathrm{~K}$ with a liquid $\mathrm{N}_{2}$ heat exchanger;

2) cooldown to $4.5 \mathrm{~K}$ with liquid $\mathrm{He}$ bath; electrical checks and validation of the quench protection heaters may be done at this stage;

3) cooldown to $1.9 \mathrm{~K}$ using a liquid He heat exchanger installed in a magnet cooling hole (see Fig. 1) and pumping on it with a $150 \mathrm{~W}, 2.7 \mathrm{~g} / \mathrm{s}$ vacuum pump to 16 mbar vapor pressure.

After cooldown and initial cold electrical checkout, including high voltage withstand tests (used to test coil-to-ground and -to-heater insulation integrity) and verifying quench protection heater operation, the quench training program at $1.9 \mathrm{~K}$ was started, with $20 \mathrm{~A} / \mathrm{s}$ ramp rate and typically $100 \mathrm{kHz}$ fast logger sampling rate. Quench tests were to be repeated until a stable quench current level was achieved, quench currents 


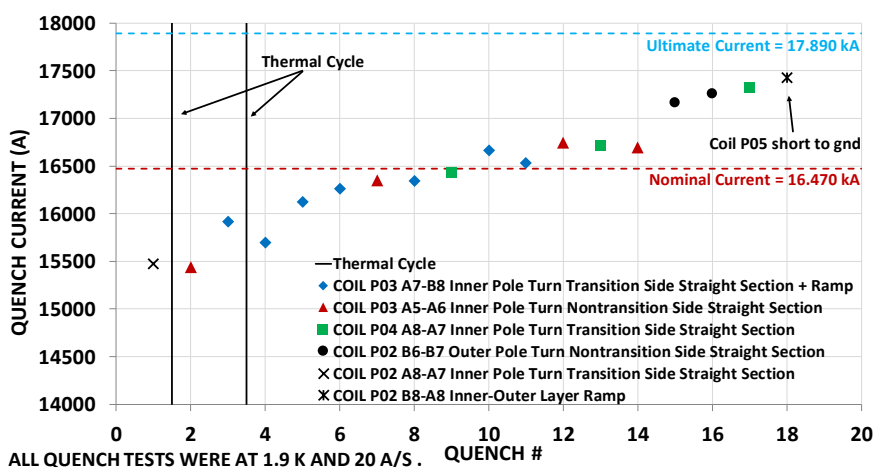

Fig. 3. MQXFAP1 quench performance plot. Testing was terminated when Coil P05 developed a short to ground.

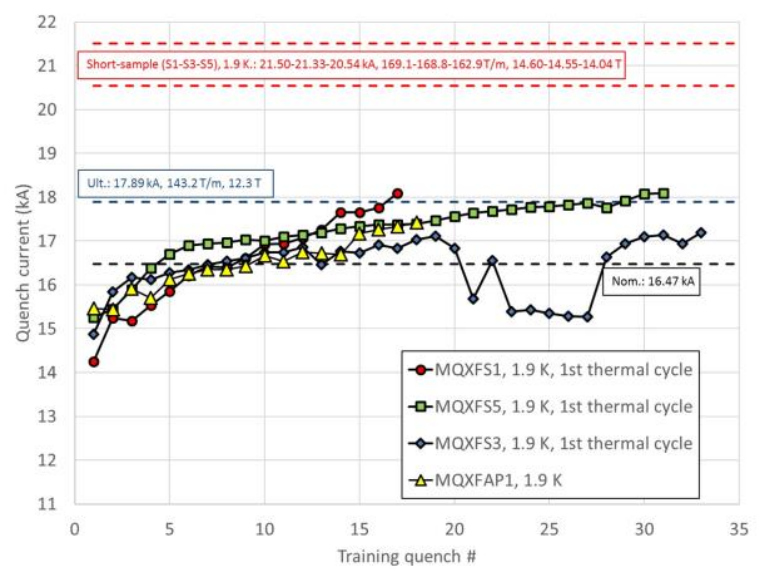

Fig. 4. Comparison of short and long MQXFA magnets training performance. MQXFAP1 training was similar to the short prototypes. This plot is courtesy of G. Ambrosio, FNAL.

reached above the ultimate current and could be held in stable operation, or quench currents were low or erratic and there was no training. If the latter case, quenches at $4.5 \mathrm{~K}$ were also done to help determine possible mechanisms, such as conductor damage, which will usually result in temperature dependence. Quench voltage tap and other signals were analyzed to look for artifacts, to verify that a quench event was not a spurious QD trip, to determine quench start location, and to calculate the quench integral to estimate quench hot spot temperature to determine if it was below the allowed maximum value.

\section{QUENCH TRAINING RESULTS}

For the first long prototype MQXFAP1, the training history is plotted in Fig. 3, and it shows that the training did follow a mostly upward trend and in fact was similar to the short models, as seen in Fig. 4. Most quenches in MQXFAP1 were in the inner layer pole turns, as is expected for a nominally training magnet. The first two quenches, each with stored energy of 3.8 MJ, resulted in the relief system burst disc rupturing and causing the escape of the He gas from the test cryostat. In each case, a thermal cycle was done and changes were made to mitigate this issue; after the second thermal cycle, when an upgraded relief valve assembly was installed, burst disc ruptures were eliminated, though some $\mathrm{He}$ gas was still being lost due to opening of the reliefs [5], [6]. As seen in Table 1, energies of up to $5.5 \mathrm{MJ}$ were possible for these tests, so further up- grades to the He gas recovery system were needed. Since this magnet test, further upgrades to the gas recovery system were made, which included addition of warm and cold buffer tanks, and this has eliminated the opening of the relief valves [5], [6].

As can be seen, in addition to a typical training curve, there was also exhibited memory of the training after each thermal cycle. This test also provided several milestones. The last 5 quenches were done with the CLIQ system added, the first such application on a long MQXFA magnet. Quench integral values decreased from $\sim 29(270 \mathrm{~K})$ to $\sim 25$ MIIts $(220 \mathrm{~K})$, as expected. Also, the last 4 quenches were done without the inner layer protection heaters, and MIIts values were not affect-

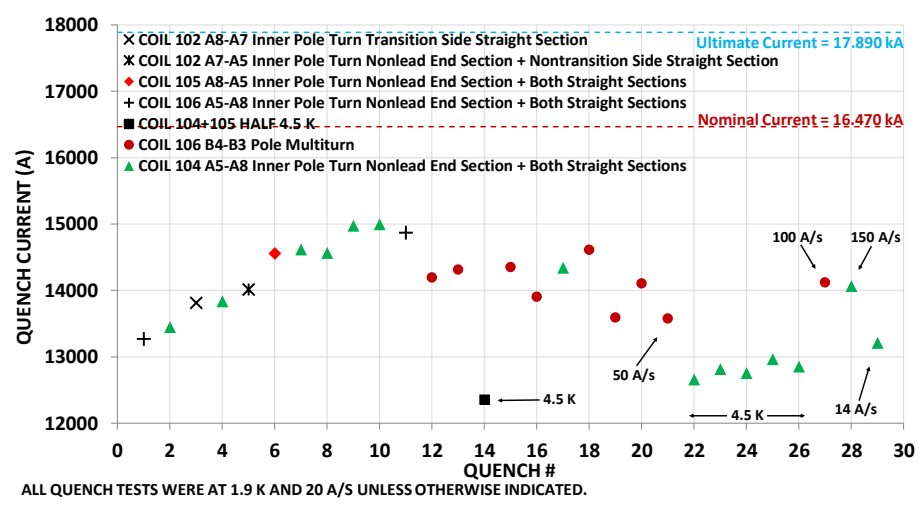

Fig. 5. MQXFAP2 quench performance plot. Quench currents were low and became erratic, though temperature dependence was exhibited.

ed. This was an important result, as inner layer heaters are no longer to be used in MQXF magnets due to bonding issues.

Unfortunately, MQXFAP1 testing ended with Quench 18, when Coil P05 developed a short to ground. High voltage withstand tests involving this coil had been problematic starting with the first cooldown, and later at the first warmup, when Coil P05 outer layer, low field heater started exhibiting a short to the coil. The Coil P05 issue is attributed to a combination of the following causes: 1) non-conforming impregnation of Coil P05, and 2) high voltage withstand tests that were done at room temperature after the testing at $1.9 \mathrm{~K}$, with the possibility of trapped He gas in the coils. After testing was finished, Coil P05 was subsequently replaced with a new $4.0 \mathrm{~m}$ coil (P06) for re-test of the magnet later as MQXFAP1b.

The quench current plot for MQXFAP2 is shown in Fig. 5, and it is seen that the magnet did not demonstrate training, starting at an atypically low first quench and remaining at currents lower than usual, not even reaching the nominal value of $16.470 \mathrm{kA}$, and quench currents eventually became erratic in nature. Quenches occurred in all four coils, but starting with Quench 7, all quenches were in Coils 104 and 106.

For all coils, most of the taps A6, A7 and B5, B6, which bracket the nonlead end sections in the inner and outer layer pole turns, respectively (see Fig. 2), opened up during quenches. Because most pole turn nonlead end voltage taps were lost, quench localization for inner pole turn quenches was compromised. However, the quenching segments with the remaining working taps did always include the nonlead end, and quench antenna data analysis [11] showed that most inner layer quenches originated in or near the nonlead end. For the 


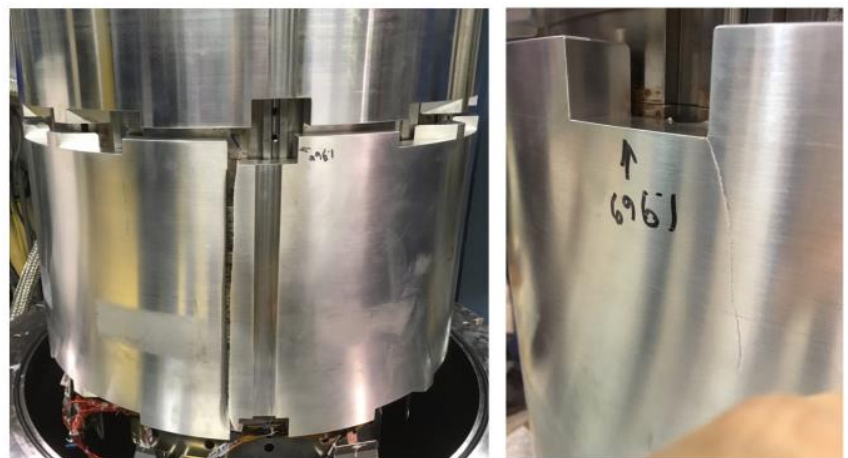

Fig. 6. MQXFAP2 nonlead end shell cracks, which all originated at corners of the alignment cut-outs. Photo is courtesy of D. Cheng, LBNL.

many outer layer multiturn quenches, however, it was not possible to localize because the antenna is installed in the magnet bore and is too far from the outer layer to sense the signals. For many quenches, voltage signals exhibited precursor spikes $0-8 \mathrm{~ms}$ prior to the quench start. The presence of precursor spikes and the erratic nature of the quench currents imply mechanical motion, but it can also be seen in the quench plot that quench currents were affected by changes in temperature, and this implies conductor damage. Quench integral values ranged from $25-28$ MIIts $(\sim 170-\sim 200 \mathrm{~K})$, which were well within the target of $250 \mathrm{~K}$ maximum for training quenches.

Testing was finally ended due to the lack of training. After warmup, it was seen that the nonlead end aluminum shell had developed a large crack along the axial direction, along with other smaller cracks starting to propagate. (See Fig. 6.) This observation agrees with the nonlead end quench locations and the loss of taps at that end. All cracks originated at corners of the alignment cut-outs; these corners are locations of concentrated stress. It was later shown that the radius of curvature of the nonlead end shell cut-out corners was too small [12].

After the Coil P05 ground short which ended the training program of MQXFAP1, P05 was replaced by a newly wound $4.0 \mathrm{~m}$ long coil, denoted P06, and the magnet, now designated MQXFAP1b, was retested. Fig. 7 shows the quench current history, and as can be seen, the first 3 quenches were in the new, untested P06, and it trained normally to nominal operating current; locations were in the inner layer pole turn, as expected. Other than one more quench in P06, which continued its training, the rest of the quenches were in the Coil P03 inner layer, somewhere in the two multiturn blocks (see Fig 2); unfortunately, tap A3 in P03 was open so the resulting section comprised all the turns other than the pole turn. Quench antenna data showed that quench locations varied axially, ranging from lead end to nonlead end and in between [13].

Quench currents 8-18 were highly erratic, implying mechanical motion. Starting with Quench 19, quench currents were stable but low, and were affected by both ramp rate and temperature, implying conductor damage. These results suggest that motion of the coil during earlier quenches might have caused damage to the conductor, as seen in the behavior of the later quenches. Since Coil P03 was now limiting the performance of the magnet, testing was stopped so that the issue could be investigated. MQXFAP1b will be disassembled and

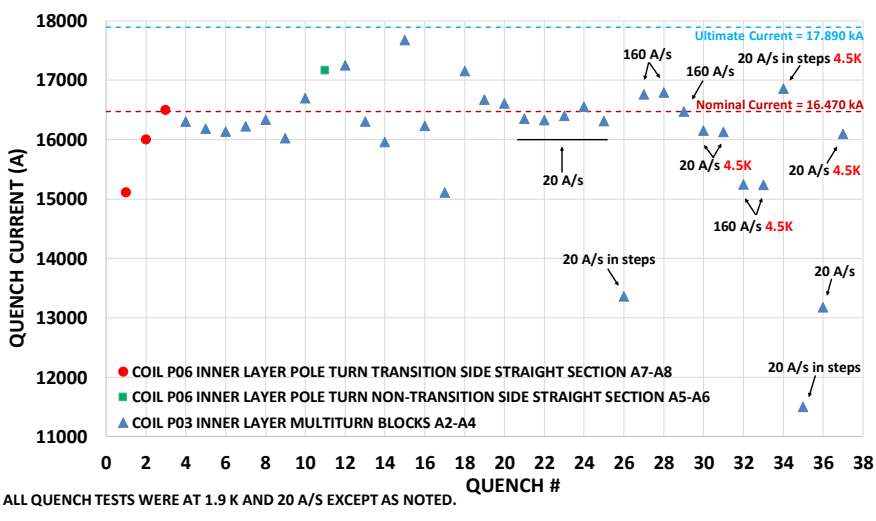

Fig. 7. MQXFAP1b quench performance plot.

Coil P03 will be inspected. Epoxy failure due to impregnation issues is suspected.

\section{LESSONS LEARNED AND MITIGATIONS}

1) MQXFAP1 Coil P05 failure:

a. The supplier of the insulation between heaters and coil has been changed.

b. The coil impregnation process has been upgraded and is still being investigated.

c. After initial cold high voltage withstand tests and exposure to He, warm high voltage target values are reduced to safer levels, a factor of 5 less than initial cold [14].

2) MQXFAP2 Nonlead End Shell failure:

a. Heat treatment of the aluminum used in the failed shell will no longer be used.

b. Pole key gap is increased to reduce shell stresses [7].

b. Shell alignment cut-outs are now to have $10-15 \mathrm{~mm}$ radius at the corners to minimize stress there [7], [12].

3) MQXFAP1b Coil P03 failure:

a. Coil P03 is still under investigation.

b. Epoxy failure is suspected.

c. Pre-load sequence has been changed [7].

\section{CONCLUSIONS}

Two MQXFA long prototypes have been tested in three tests: MQXFAP1, MQXFAP2, and MQXFAP1b. In each case, there was a specific failure which pointed to an issue which has been addressed or is under investigation. MQXFAP1 training and early MQXFAP $1 b$ training does validate the long magnet structural design, but specific issues uncovered by the testing are being addressed before the test of MQXFA03, the first pre-series MQXFA magnet. Also, during the testing, various modifications to the test facility were implemented to increase the efficiency of the test procedures, to accommodate the high quench energies for the He gas recovery system, and to meet the testing requirements of the Hi-Lumi program.

\section{ACKNOWLEDGMENT}

The authors thank W. McKeon, S. Dimiauta, D. Sullivan, and J. D'Ambra for their invaluable work during testing. 


\section{REFERENCES}

[1] P. Ferracin, et al, "The HL-LHC Low- $\beta$ Quadrupole Magnet MQXF: From Short Models to Long Prototypes," IEEE Trans. Appl. Supercon. vol. 29, no. 5, Aug. 2019 Art. no. 4001309.

[2] R. Carcagno, G. Ambrosio, and G. Apollinari, "MQXFA Magnet Functional Requirements Specification," US-HiLumi-doc-36, July 11, 2017.

[3] S. Feher and G. Ambrosio, "Test Stand Functional Requirements for Testing MQXFA Magnets and Q1/Q3 Cryostat Assemblies," USHiLumi-doc-1109, June 21, 2018.

[4] J. Muratore, M. Anerella, P. Joshi, P. Kovach, A. Marone, P. Wanderer, "Design and fabrication of the $1.9 \mathrm{~K}$ magnet test facility at BNL and test of the first 4-m-long MQXF coil," IEEE Trans. Appl. Supercond., vol. 28, no. 3, Apr. 2018.

[5] J. Muratore, "MQXFAP2 Test Status Update," $8^{\text {th }}$ HL-LHC Collaboration Meeting CERN Oct. 15-18, 2018. [Online]: Available: https://indico.cern.ch/event/742082/contributions/3141439/

[6] J. Muratore, "Q1/Q3 Cryo-assemblies Fab 302.4.01 Magnets Vertical Test at BNL," HL-LHC AUP CD-2/3b DOE/SC Review, Dec 11-13, 2018. [Online]: Available: https://indico.fnal.gov/event/19016/

[7] D.W. Cheng, et al, "Mechanical performance of the first two prototype $4.5 \mathrm{~m}$ long Nb3Sn low- $\beta$ quadrupole magnets for the Hi-Lumi LHC Upgrade," IEEE Trans. Appl. Supercond., vol. 30, no. 4, June 2020.

[8] H. Song, et al, "Vertical Magnetic Measurements of the First FullLength Prototype MQXFAP2 Quadrupole for the LHC Hi-Lumi Accelerator Upgrade Project," IEEE Trans. Appl. Supercond., vol. 29, no. 5, pp. 1-7, Aug. 2019, Art no. 4004707.

[9] H. Song, et al, "Vertical and High Current Magnetic Field Measurements of Full-Length MQXFAP Quadrupoles at Cryogenic Temperatures for Hi-Lumi LHC \& PCB Rotating Coil Magnetic Field Measurement at BNL," IEEE Trans. Appl. Supercond., vol. 30, no. 4, June 2020.

[10] E. Ravaioli, “CLIQ," Ph.D. dissertation, Dept. Sci. Tech., Univ. Twente, Enschede, The Netherlands, 2015.

[11] M. Marchevsky, unpublished data, Oct. 2018.

[12] H. Pan, et al, "Fracture Failure Analysis for MQXF Magnet Aluminum Shells," IEEE Trans. Appl. Supercond., vol. 30, no. 4, June 2020.

[13] M. Marchevsky, AUP MQXFA Weekly Meeting June 24, 2019. [Online]. Available: https://indico.fnal.gov/event/21082/

[14] V. Marinozzi, et al, "Analysis of the heater-to-coil insulation in MQXF coils," IEEE Trans. Appl. Supercond., vol. 30, no. 4, June 2020. 